\title{
The framing effect and risky decisions: Examining cognitive functions with fMRI
}

\author{
Cleotilde Gonzalez ${ }^{\mathrm{a}, *}$, Jason Dana ${ }^{\mathrm{a}}$, \\ Hideya Koshino ${ }^{\mathrm{b}}$, Marcel Just ${ }^{\mathrm{c}}$ \\ ${ }^{a}$ Social and Decision Sciences, Carnegie Mellon University, Pittsburgh, PA 15213, USA \\ b Department of Psychology, California State University, San Bernardino, CA 92407, USA \\ c Center for Cognitive Brain Imaging, Department of Psychology, Carnegie Mellon University, \\ Pittsburgh, PA 15213, USA
}

Received 7 November 2003; received in revised form 17 August 2004; accepted 23 August 2004 Available online 5 November 2004

\begin{abstract}
The "framing effect" is observed when the description of options in terms of gains (positive frame) rather than losses (negative frame) elicits systematically different choices. Few theories explain the framing effect by using cognitive information-processing principles. In this paper we present an explanatory theory based on the cost-benefit tradeoffs described in contingent behavior. This theory proposes that individuals examining various alternatives try to determine how to make a good decision while expending minimal cognitive effort. For this study, we used brain activation functional magnetic resonance imaging (fMRI) to evaluate individuals that we asked to choose between one certain alternative and one risky alternative in response to problems framed as gains or losses. Our results indicate that the cognitive effort required to select a sure gain was considerably lower than the cognitive effort required to choose a risky gain. Conversely, the cognitive effort expended in choosing a sure loss was equal to the cognitive effort expended in choosing a risky loss. fMRI revealed that the cognitive functions used by the decision makers in this study were localized in the prefrontal and parietal cortices of the brain, a finding that suggests the involvement of working memory and imagery in the selection process.
\end{abstract}

(c) 2004 Elsevier B.V. All rights reserved.

\footnotetext{
${ }^{*}$ Corresponding author. Tel.: +1 412268 6242; fax: +1 4122686938.

E-mail address: conzalez@andrew.cmu.edu (C. Gonzalez).
} 
JEL classification: D8; D80; D81

PsycINFO classification: 2500; 2340

Keywords: Framing effect; Decision-making; Risk; fMRI

\section{Introduction}

The "framing effect" is observed when a decision maker's risk tolerance (as implied by their choices) is dependent upon how a set of options is described. Specifically, people's choices when faced with consequentially identical decision problems framed positively (in terms of gains) versus negatively (in terms of losses) are often contradictory. The "Asian disease problem" described by Tversky and Kahneman (1981) is a classic example of the framing effect. Decision makers were asked to choose between a certain (i.e., sure) or a probabilistic (i.e., risky) option to save lives (positive frame) or minimize deaths (negative frame) from an unusual disease:

Imagine that the United States is preparing for an outbreak of an unusual Asian disease that is expected to kill 600 people. Two alternative programs to combat the disease have been proposed. Scientific estimates of the consequences of the programs are as follows:

Positive frame:

If Program $A$ is adopted, exactly 200 people will be saved.

If Program B is adopted, there is a 1 in 3 probability that all 600 people will be saved and $a 2$ in 3 probability that no people will be saved.

Negative frame:

If Program $C$ is adopted, exactly 400 people will die.

If Program $D$ is adopted, there is a 1 in 3 probability that nobody will die and a 2 in 3 probability that all 600 will die.

Researchers who examine responses to problems of this sort generally find that negatively framed problems primarily elicit risky responses while positively framed problems primarily elicit more sure (i.e., less risky) responses. After consideration of the above example, most people chose options A and D, despite the fact that in terms of consequences, these choices are contradictory (A is equivalent to C, as B is to D). People appear to exhibit a general tendency to be risk seeking when confronted with negatively framed problems and risk averse when presented with positively framed problems.

In the past 30 years, hundreds of empirical studies ${ }^{1}$ have been conducted to demonstrate and investigate the framing effect in many different contexts (Kuhberger, 1998). Similarly, many theories have been developed to explain human behavior

\footnotetext{
${ }^{1}$ An average of 15 studies per year since the mid-80s (Kuhberger, 1998).
} 
based on assessments of gains and losses (Kuhberger, 1997). Despite all this research, cognitive theories designed to evaluate the processing demands and the kind of cognitive functions involved in the framing effect are very scarce. In this paper we propose a cognitive model based on cognitive cost-benefit tradeoff theory (Payne, Bettman, \& Johnson, 1993). In the proposed model, costs and benefits interplay with cognitive and affective processes. In addition, we test this model by using functional magnetic resonance imaging (fMRI), a technique that helps us measure the cognitive effort involved in making a choice.

\subsection{Background: Framing effect theories}

Multiple theories have been devised to explain the framing effect (Kuhberger, 1998). These are broadly divided into formal, cognitive and motivational theories.

Prospect Theory, the most well-known formal theory, explains the framing effect in terms of the value function for goods perceived as gains and losses from a reference point (Kahneman \& Miller, 1986b; Kahneman \& Tversky, 1979). Whether an outcome is perceived as a gain or a loss depends upon the individual's reference point, which is usually taken to the "status quo" asset level at the time of the choice. The value function yields the preference value assigned to outcomes, and is concave for gains, convex for losses, and steeper for losses than for gains. This functional form implies that decision makers are more sensitive to losses than to gains and exhibit diminishing marginal sensitivity to both. Therefore, people will tend to opt for a sure alternative perceived as a gain rather than for a risky alternative of equal expected value, while the converse will hold true for perceived losses.

Cognitive theories are designed to determine the cognitive processing involved in weighting gains and losses. For example, the fuzzy-trace theory proposes that the framing effect is the result of superficial and simplified processing of information (Reyna \& Brainerd, 1991). To evaluate this theory, researchers suggested and tested mechanisms by which decision makers might simplify framing problems by reasoning in qualitative patterns rather than in probabilistic and numerical patterns. The findings suggest that participants follow the path of greatest simplicity by using simplification mechanisms to reduce cognitive demands.

More comprehensively, cognitive cost-benefit tradeoff theory defines choice as a result of a compromise between the desire to make a correct decision and the desire to minimize effort (Payne et al., 1993). This theory holds that individuals initially peruse the available alternatives to determine if they can make a good decision and expend minimal cognitive effort. They only commit to a more complicated cognitive effort if they cannot fulfill their desire to arrive at a good decision by embracing a simpler alternative. Although this is an appealing explanation of the framing effect, this model ignores affective processes that should play an important role in determining what constitutes a good decision.

Motivational theories explain the framing effect as a consequence of hedonic forces, such as the fears and wishes of an individual (Lopes, 1987; Maule, 1995). According to these models, decision makers assign stronger value to feelings of 
displeasure than to feelings of pleasure, and this disparity increases proportionately with the amount of gain or loss involved in a decision (Mellers, Schwartz, \& Ritov, 1999). In other words, like Prospect Theory's assumption that losses loom larger than equivalent gains, motivational models are based on the claim that the emotions evoked by the losses generally are greater than those evoked by gains.

We will present a theory which brings cognitive and affective theories together. This new model proposes an interplay of the cognitive cost-benefit tradeoff and the motivational models to explain the choice process that leads to the framing effect and perhaps to other decisions under risk and uncertainty. The model is additionally motivated by recent findings from neuroscience that may prove relevant to economics and decision making.

\subsection{A cognitive-affective tradeoff model}

We propose that the framing effect occurs due to a tradeoff between the cognitive effort required to calculate expected values of an alternative (if processing is costly, people are less likely to choose the stimulus) and the affective value of the alternative (if the outcome produces a feeling of displeasure, people are less likely to choose the stimulus).

In a positive frame, the compromise between arriving at a good decision and minimizing cognitive effort is easy to achieve; for example, selecting the option in which "200 people will be saved" feels "correct" in an emotional sense and is effortless (i.e., no calculations are necessary). If the decision maker expends the cognitive effort required to analyze the more risky option, this alternative also will feel emotionally correct and thus appear viable. In contrast, such compromises are more difficult to attain in the negative frame. Although the option in which " 400 people will die" is easy to analyze the relatively bad outcome makes it a less than ideal choice (i.e., strong feeling of displeasure). Thus when selecting among options presented in a negative frame, individuals are more willing to undertake the cognitive effort demanded to assess the more risky option because they are more focused on improving the outcome.

Payne et al. (1993) have published findings showing that individuals take longer to make decisions when the options are framed as losses rather than gains. But does that mean that cognitive effort is greater in the negative than in the positive frame or does that mean that the affective cost is larger? And how would this cost vary for different risk levels? We propose that the costs and benefits involved in this kind of choices are of two types - cognitive and affective - and that both play a role in the framing effect. On the one hand, the cognitive effort involved in calculating an expected value is larger in risky than in certain choices and on the other hand, the affective cost is higher for losses than gains.

Neuroscience can help disentangle these issues, as it is possible to measure the amount and strength of processing involved in making choices. A better understanding of the physical mechanisms by which human decisions are made is of growing interest for both economists and neuroscientists (Glimcher, 2003). fMRI studies suggest that cognition and emotion integrate in the prefrontal cortex (PFC) of the brain 
when making simple choices (Gray, Braver, \& Raichle, 2002). ${ }^{2}$ Independently, the PFC has been associated with both affective processes and with the processing of risk and uncertainty.

Damasio and colleagues have documented the role of the PFC in decision making (Bechara, Damasio, \& Damasio, 2000). The most general conclusion from these studies is that emotional defects produce impaired decision making and that a section of the PFC known as the ventromedial prefrontal cortex (BA 11, 12, 13, and 25 ) is particularly important to decision making. Their methodology often involves patients with lesions in the PFC as well as healthy participants (Bechara, Damasio, Tranel, \& Damasio, 1997).

The task used in their studies involves two decks of cards that produce negative expected values in the long run but have extreme gains and losses and two other decks of cards that produce positive expected values with less extreme outcomes. The main finding is that PFC patients return rapidly to the less advantageous decks after suffering a loss, although the immediate emotional reaction (measured by skin conductance) to losses is the same as in normal subjects. They explain the results with the somatic-marker hypothesis which poses that decision making is dependent on emotional processes. As suggested by their results, damage in the ventromedial prefrontal cortex precludes the use of somatic signals necessary to guide decision making in an advantageous direction (Bechara et al., 2000).

In addition to affective processes, the PFC has been associated with processing risk and uncertainty in decision making. Different versions of a guessing task have been used to examine risky decisions (Elliott, Rees, \& Dolan, 1999; Paulus et al., 2001; Rogers et al., 1999). For example, activity in the PFC increases during individuals' consideration of uncertain rather than certain conditions in two-choice prediction tasks that have no "correct" response (Elliott et al., 1999; Paulus et al., 2001; Rogers et al., 1999). Conditions with uncertain outcomes elicit more activity in the prefrontal and parietal cortices (BA 10, 7, and 40) than do those with assured outcomes (Paulus et al., 2001). Furthermore, the PFC has also been associated with differential activation in alternatives involving monetary rewards and penalties (Delgado, Nystrom, Fissel, Noll, \& Fiez, 2000; Elliott, Friston, \& Dolan, 2000; Knutson, Fong, Adams, Varner, \& Hommer, 2001; O’Doherty, Kringelbach, Rolls, Hornak, \& Andrews, 2001). PFC activity continues for a longer period of time after a reward feedback than after a punishment feedback (Delgado et al., 2000). An fMRI study of the Prospect Theory also addresses the anticipation and receipt of monetary rewards and penalties (Breiter, Aharon, Kahneman, Dale, \& Shizgal, 2001). When expectations of and responses to monetary gains and losses were mapped to brain activity, higher PFC (BA 10) activity was found in response to the size of the rewards or penalties than to whether they were gains or losses.

In summary, this research proposes a two-pronged explanation to describe the posited connection between the PFC and the formulation of responses to positively

\footnotetext{
${ }^{2}$ See Appendix A for a brief introduction to the brain cortex, the main brain regions and the more specific functional areas.
} 
and negatively framed problems. First, the desire to arrive at a good decision can be heavily charged with emotions in people attempting to do well and avoid bad outcomes. Second, the desire to minimize cognitive effort can lead to activation in the PFC as individuals determine the expected value of various alternatives. Thus we expect to observe an interaction between the activations associated with frame and risk of the selected alternative. If cognitive and affective processes interplay to produce different choices in positive and negative frames, we should be able to see differential brain activity due to both the frame and the risk of the outcome. The negative frame would produce more feelings of displeasure than the positive frame resulting in more brain activity in the PFC; at the same time risky alternatives would be more cognitively difficult due to the calculation of an expected value, producing higher PFC activation than the certain choices.

\section{Methods}

\subsection{Participants}

Fifteen healthy, right-handed college student volunteers ( 5 females, 10 males) gave signed, informed consent to participate on this study, which was approved by the University of Pittsburgh and the Carnegie Mellon Institutional Review Boards. They were paid a standard amount of 30 dollars including training time in the lab and time in the MRI scanner.

Participants were familiarized with the scanner, the fMRI procedure, and the risk task by responding (while in the scanner) to four problems in the same format as the ones they would receive during the study. The data for three participants were discarded due to the participants' excessive head motion during testing. Additionally, the data for two other participants who had zero responses to one of the conditions were discarded.

\subsection{Experimental design}

Participants were asked to make 20 choices in response to 10 different problems, each of which was presented in both positive and negative frames. A meta-analysis of previously published articles was used to select stimulus items that have been documented to clearly demonstrate the influence of framing on risky decision making (Kuhberger, 1998). The problems were in the format of the well-known (and aforementioned) Asian disease problem, with two possible responses: one certain outcome and one risky outcome. The positive and negative articulations of each problem were presented randomly with one caveat: the two framings of each problem always were separated by at least one other problem. The order of the certain and risky options was counter-balanced within both framing conditions.

Choice trials were presented one at a time and were separated by rest periods to allow for event-related analysis. Each trial began with the 10 -s written presentation of a problem portrayed on a display in the scanner. The two choice options then 
were added to the display, and the cumulative display remained visible for $18 \mathrm{~s} .^{3}$ Participants were instructed to make a decision and wait for the next screen to appear. Choices were entered by pushing one of the two buttons on a control box. Participants could enter their choices at any time during the 18-s response period. A message indicating that time was about to expire was displayed $2 \mathrm{~s}$ before the choice interval elapsed. Each trial was followed by a rest period of $12 \mathrm{~s}$ to allow the hemodynamic response to diminish. Five fixation periods of $25 \mathrm{~s}$ were interspersed and distributed evenly throughout the acquisition to obtain a control base-line measure of brain activation with which to compare the experimental conditions. During the rest and fixation periods, an "X" was displayed at approximately center screen.

\subsection{MRI acquisition parameters}

The study was conducted in a GE 3.0 Tesla scanner at the Magnetic Resonance Research Center of the University of Pittsburgh Medical Center. The fMRI data for each participant were acquired in a single run consisting of 1030 images per volume. The run lasted approximately $18 \mathrm{~min}$, during which the participant solved a total of 20 problems, each presented for a total of $28 \mathrm{~s}$, with a data sampling rate of $1 \mathrm{~Hz}(\mathrm{TR}=1 \mathrm{~s})$. Sixteen functional slice images were acquired in an oblique-axial plane as shown in Fig. 1. The pitch angle of the images ranged from $15^{\circ}$ to $28^{\circ}$, $\mathrm{TR}=1000 \mathrm{~ms}, \mathrm{TE}=18 \mathrm{~ms}$, flip angle $=70^{\circ}$, and a $3.125-\mathrm{mm} \times 3.125-\mathrm{mm} \times 5-\mathrm{mm}$ matrix voxel size with a 1-mm gap.

A 124-slice axial T1-weighted 3D Spoiled GRASS structural volume scan with $\mathrm{TR}=25 \mathrm{~ms}, \mathrm{TE}=4 \mathrm{~ms}$, flip angle $=40, \mathrm{FOV}=24 \mathrm{~cm}$ and a $256 \times 192$ matrix size, was acquired for each participant after the functional data were acquired. This scan was used in parcellating the functional images into anatomically predefined regions of interest (see below).

\section{4. fMRI data analysis}

Image preprocessing was performed to correct for in-plane head motion and signal drift using FIASCO (Eddy, Fitzgerald, Genovese, Mockus, \& Noll, 1996; further description of the tools available at www.stat.cmu.edu/ fiasco/). The fMRI data obtained during the first $6 \mathrm{~s}$ after stimulus presentation were discarded to accommodate the rise of the hemodynamic (BOLD) response to its peak level (Bandettini, Wong, Hinks, Tokofsky, \& Hyde, 1992).

To compare the volume of activation across the experimental conditions, a priori regions of interest (ROIs) were defined for each participant. The ROIs were defined using the parcellation method originally described by Rademacher, Galaburda, Kennedy, Filipek, and Caviness (1992) and subsequently refined by Caviness, Kennedy, Bates, and Makris (1996). Because each individual cortical anatomy is different, the ROIs were drawn on the structural images of each

\footnotetext{
${ }^{3}$ The duration of the two presentation intervals was determined by conducting pilot studies.
} 


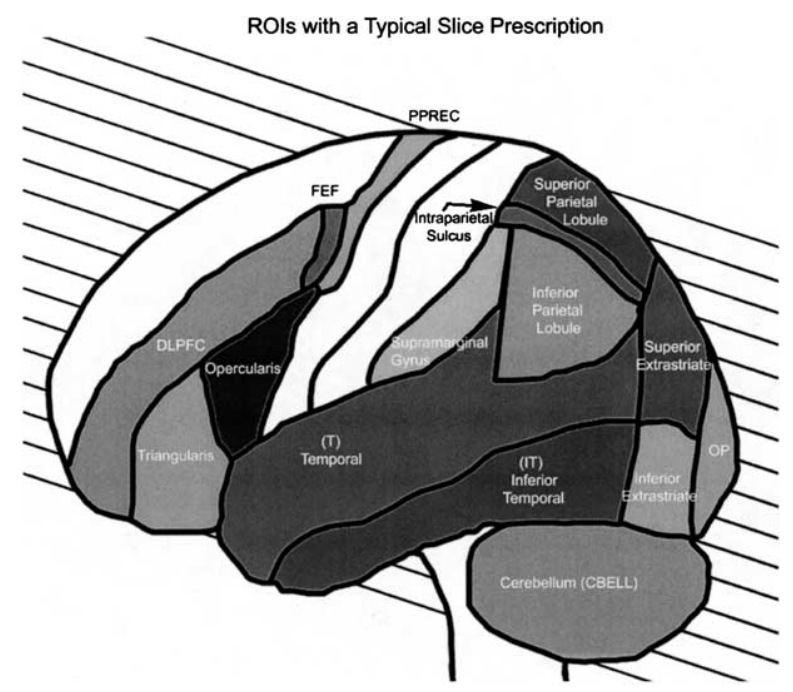

Fig. 1. Regions of interest (ROIs) with slice prescription.

participant to precisely target the anatomical regions of interest (the surfaces of the ROIs are shown in Fig. 1). The method has been consistently and successfully used in many previous studies to compare the amount of activation in a given region across conditions (e.g., Just et al., 2001; Newman, Just, \& Carpenter, 2002). The ROIs abbreviations as used in this study and their corresponding Talairach coordinates are listed in Table 1.

To investigate the patterns of activation according to the response variable, the activation data was sorted with respect to both the frame and the response to form four groups: positive-certain, positive-risky, negative-certain, and negative-risky. The number of problems was equalized per subject to enable analysis of the same number of images per condition. For each individual, the problems per condition were chosen to match the number of problems in the individual with the minimum number.

Activation was quantified for each of these four conditions using FIASCO. First, a $t$ map was constructed by computing the difference between each voxel's activation in each condition and the base-line measure of activation (the fixation period). Voxels whose signal change exceeded base-line by a $t$ value $>5.5$ were considered active. This threshold yielded an alpha-level of less than 0.02 after Bonferroni correction for 16,000 voxels in all regions of interest.

Once the number of activated voxels was calculated, the mean percent increase in the amplitude of activation relative to the base line condition was calculated for those voxels. These two values, the number of voxels and the change in activation were used to calculate the dependent measure in this study (sum of signal intensity, SSI). SSI was calculated by adding the percentage change in signal intensity for each voxel activated in a particular condition and comparing this integral measure across 
Table 1

ROIs and Talairach coordinates

\begin{tabular}{|c|c|c|c|c|}
\hline \multirow[t]{2}{*}{ ROI } & \multirow[t]{2}{*}{ Description } & \multicolumn{3}{|c|}{ Talairach coordinates } \\
\hline & & $X$ & $Y$ & $Z$ \\
\hline \multicolumn{5}{|l|}{ Frontal } \\
\hline LDLPFC & Left-dorsolateral prefrontal cortex & -30.8 & 27.9 & 37.9 \\
\hline LFEF & Left-frontal eye field & -43.1 & 3 & 45.3 \\
\hline LOPER & Left-pars opercularis & -44.8 & 12.9 & 25 \\
\hline LPOSTPRECEN & Left-posterior precentral sulcus & -41.2 & -4.8 & 43.4 \\
\hline LTRIA & Left-pars triangularis & -41.1 & 22.5 & 15.4 \\
\hline RDLPFC & Right-dorsolateral prefrontal cortex & 35.7 & 21.7 & 40.6 \\
\hline RFEF & Right-frontal eye field & 41.3 & 9.1 & 42.2 \\
\hline ROPER & Right-pars opercularis & 47.1 & 13 & 19.3 \\
\hline RPOSTPRECEN & Right-posterior precentral sulcus & 38.8 & -1.3 & 48 \\
\hline RTRIA & Right-pars triangularis & 42.9 & 25.2 & 20.3 \\
\hline SMA & Supplementary motor area & -0.1 & -5.6 & 68.2 \\
\hline SMFP & Superior medial frontal/paracingulate & -0.2 & 22.4 & 54.4 \\
\hline \multicolumn{5}{|l|}{ Parietal } \\
\hline LIPL & Left-inferior parietal & -44 & -58.4 & 28.8 \\
\hline LIPS & Left-intraparietal sulcus & -28.3 & -65.4 & 46.7 \\
\hline LSGA & Left-supramarginal gyrus & -52.5 & -37.6 & 37.8 \\
\hline RIPL & Right-inferior parietal & 46.8 & -50.5 & 34.7 \\
\hline RIPS & Right-intraparietal sulcus & 31.3 & -62.1 & 45 \\
\hline RSGA & Right-supramarginal gyrus & 51.2 & -25.8 & 46.5 \\
\hline \multicolumn{5}{|l|}{ Temporal } \\
\hline LIT & Left-inferior temporal & -42.5 & -61.3 & 2 \\
\hline $\mathrm{LT}$ & Left-temporal & -55.6 & -32.4 & 7.5 \\
\hline RIT & Right-inferior temporal & 44.4 & -62.3 & 2.3 \\
\hline RT & Right-temporal & 52.2 & -32.1 & 8.3 \\
\hline \multicolumn{5}{|l|}{ Occipital } \\
\hline CALC & Calcarine fissure & 4.3 & -77.1 & 8.9 \\
\hline LIES & Left-inferior extrastriate & -21.2 & -75.8 & -1.6 \\
\hline LSES & Left-superior extrastriate & -16.2 & -82.6 & 36.8 \\
\hline OP & Occipital pole & 3.1 & -90.7 & 8.9 \\
\hline RIES & Right-inferior extrastriate & 24.6 & -77.9 & 1.1 \\
\hline RSES & Right-superior extrastriate & 23.9 & -83.2 & 27.9 \\
\hline
\end{tabular}

conditions. This measure, first reported by Xiong, Rao, Gao, Woldor, and Fox (1998), has since been used in many brain imaging articles. All statistical analyses involved within-subjects ANOVAs with both frame and risk as within-subjects variables.

Although this study was particularly motivated to do analyses on the ROIs of the PFC there is little information on the role of other cortical components on decision making. Some studies suggest that the parietal cortex is also involved in processing risk and uncertainty (Greene, Sommerville, Nystrom, Darley, \& Cohen, 2001; Rogers et al., 1999). Thus, statistical analyses in all of the ROIs of the four lobes were performed rather than only doing these on the ROIs of the PFC. 


\section{Results}

\subsection{Behavioral results}

The distribution of choices over the positively and negatively framed problems was consistent with framing results generated in previous studies. Participants chose the certain option more often when responding to positively framed problems and the risky option more often in response to negatively framed problems (Fig. 2, top panel). The percentage of risk-seeking choices was 33\% under positive framing and $59 \%$ under negative framing $\left(\chi^{2}(1)=13.6, p<0.01\right)$. These results were obtained despite the within-subject design of the study, which might be expected to encourage consistency across responses to the same problem. Most behavioral studies employ
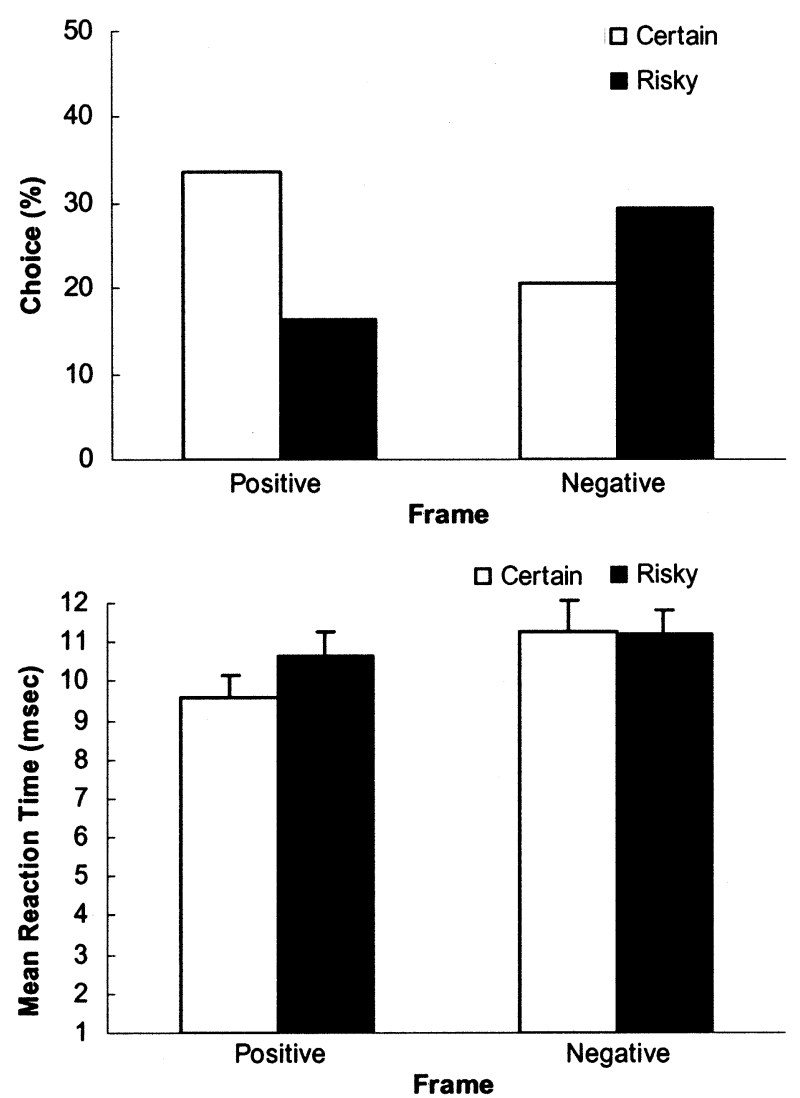

Fig. 2. Behavioral results: number of choices and reaction time. Top panel shows the mean number of certain and risky choices in response to positively and negatively framed problems. Bottom panel shows the reaction time for certain and risky choices in response to positively and negatively framed problems. Error bars represent the standard error of the mean. 
between-subject designs to eliminate the consistency problem (Levin, Gaeth, Schreiber, \& Lauriola, 2002).

Behavioral studies often do not measure reaction time because such research tends to focus on the outcomes rather than the processes involved in the framing effect. Our analyses of reaction time revealed that participants took more time to make a choice in negatively framed problems than in positively framed problems $(11,227 \mathrm{~ms}$ versus $9990 \mathrm{~ms}$, respectively; $F(1,9)=17.5, p<0.01$; Fig. 2 , bottom panel), but took approximately the same amount of time across all frames regardless of whether the participants selected the certain or the risky alternatives $(F(1,9)=1.19$, n.s.). The interaction between frame and risk was not significant $(F(1,9)=2.2$, n.s. $)$.

\section{2. fMRI results}

The main fMRI finding was that activation differed between risky and certain choices, but only for the positive and not for the negative frame. The selection of risky alternatives in positive framed problems led to higher activation in frontal, parietal and occipital areas than did the selection of certain alternatives. The level of activation was very similar for risky and certain choices in the negative frames. The effect of differential activation did not seem to be due to the frame but rather, to the risk of the alternatives chosen. Risky alternatives produced higher activation than certain alternatives in particular in frontal and parietal areas.

For completeness, in Table 2, we present the activation and ANOVA results for all of the ROIs, but the most salient entries in the table are presented in bold.

Comparisons of certain and risky choices in response to positively framed problems revealed significant differences in several ROIs (right-most column in Table 2). Higher activation levels were observed in positive-risky choices than in positive-certain choices. As shown in Table 2 this difference was significant in the RDLPFC (BA 9, middle and BA 46) and RPOSTPRECEN (BA 8) regions. In the parietal cortex, activation levels in participants making positive-risky choices were significantly higher in the LIPL (BA 40), right and left IPS (between BA 7 and 40), and RSPL (BA 7) regions. We also observed significantly higher activation levels in the right and left IES (BA 19) regions of the occipital cortex when participants made positive-risky choices. Fig. 3 shows these differences between certain and risky choices in a graph.

The same analyses indicated non-significant differences in all ROIs $(p<0.05)$ for negatively framed choices.

Fig. 4 depicts the average activation patterns. As indicated, selection of the risky choices resulted in significantly more activation in the frontal and parietal areas compared to the selection of certain choices. This difference is clear only in the positive frame. The activation does not differ between certain and risky choices in the negative frame.

In general, activation resulting from participants' selection of risky versus certain choices varied in frontal and parietal areas. The selection of risky rather than certain choices led to higher activation levels in the right dorsolateral prefrontal 
Table 2

Sum of the change signal intensity (SSI) per ROI and condition

\begin{tabular}{|c|c|c|c|c|c|c|c|c|}
\hline \multirow[t]{2}{*}{ ROI } & \multirow[b]{2}{*}{$\begin{array}{l}\text { Positive/ } \\
\text { certain }\end{array}$} & \multirow[b]{2}{*}{$\begin{array}{l}\text { Positive/ } \\
\text { risky }\end{array}$} & \multirow[b]{2}{*}{$\begin{array}{l}\text { Negative/ } \\
\text { certain }\end{array}$} & \multirow[b]{2}{*}{$\begin{array}{l}\text { Negative/ } \\
\text { risky }\end{array}$} & \multirow{2}{*}{$\begin{array}{c}\text { Frame } \\
F(1,9)\end{array}$} & \multirow{2}{*}{$\begin{array}{l}\text { Risk } \\
\\
F(1,9)\end{array}$} & \multirow{2}{*}{$\begin{array}{l}\text { Frame } \times \\
\text { Risk } \\
\\
F(1,9)\end{array}$} & \multirow{2}{*}{$\begin{array}{l}\text { Risk in } \\
\text { positively } \\
\text { framed } \\
\text { problems } \\
E \\
F(1,9)\end{array}$} \\
\hline & & & & & & & & \\
\hline \multicolumn{9}{|l|}{ Frontal } \\
\hline LDLPFC & 11.7 & 16.8 & 18.9 & 17.4 & n.s. & n.s. & n.s. & n.s. \\
\hline LFEF & 7.9 & 12.6 & 13.0 & 15.8 & n.s. & n.s. & n.s. & n.s. \\
\hline LOPER & 6.3 & 7.3 & 8.2 & 7.4 & n.s. & n.s. & n.s. & n.s. \\
\hline LPOSTPRECEN & 4.7 & 9.9 & 8.0 & 9.4 & n.s. & n.s. & n.s. & n.s. \\
\hline LTRIA & 1.7 & 1.8 & 1.0 & 1.9 & n.s. & n.s. & n.s. & n.s. \\
\hline RDLPFC & 12.5 & 29.4 & 18.8 & 22.9 & n.s. & $3.8^{*}$ & n.s. & $7.2^{* *}$ \\
\hline RFEF & 5.5 & 11.3 & 4.6 & 5.0 & n.s. & n.s. & n.s. & n.s. \\
\hline ROPER & 0.7 & 2.5 & 2.9 & 1.1 & n.s. & n.s. & n.s. & n.s. \\
\hline RPOSTPRECEN & 3.7 & 7.7 & 5.2 & 4.0 & n.s. & n.s. & $3.9^{*}$ & $6.23^{* *}$ \\
\hline RTRIA & 0.5 & 2.5 & 1.3 & 1.2 & n.s. & n.s. & n.s. & n.s. \\
\hline SMA & 4.4 & 4.7 & 5.3 & 5.8 & n.s. & n.s. & n.s. & n.s. \\
\hline SMFP & 12.6 & 17.6 & 21.3 & 18.6 & n.s. & n.s. & n.s. & n.s. \\
\hline Sum & 72.0 & 124.1 & 108.6 & 110.5 & n.s. & $3.41^{*}$ & n.s. & n.s. \\
\hline \multicolumn{9}{|l|}{ Parietal } \\
\hline LIPL & 8.5 & 16.4 & 16.0 & 13.1 & n.s. & n.s. & $5.4^{* *}$ & $4.51^{*}$ \\
\hline LIPS & 18.4 & 38.7 & 28.1 & 32.7 & n.s. & $4.88^{*}$ & $4.0^{*}$ & $8.71^{* *}$ \\
\hline LSGA & 0.3 & 2.5 & 1.3 & 0.6 & n.s. & n.s. & n.s. & n.s. \\
\hline LSPL & 5.2 & 9.5 & 6.7 & 5.4 & n.s. & n.s. & n.s. & n.s. \\
\hline RIPL & 8.9 & 13.3 & 13.7 & 10.1 & n.s. & n.s. & n.s. & n.s. \\
\hline RIPS & 30.7 & 53.6 & 40.8 & 41.4 & n.s. & $\mathbf{8 . 8 8}^{* *}$ & n.s. & $15.69^{* * *}$ \\
\hline RSGA & 0.0 & 0.7 & 0.5 & 0.4 & n.s. & n.s. & n.s. & n.s. \\
\hline RSPL & 10.8 & 21.8 & 13.3 & 13.6 & n.s. & n.s. & n.s. & $6.61^{* *}$ \\
\hline Sum & 82.9 & 156.5 & 120.5 & 117.2 & n.s. & $3.96^{*}$ & n.s. & $10.60^{*}$ \\
\hline \multicolumn{9}{|l|}{ Temporal } \\
\hline LIT & 3.3 & 4.0 & 7.0 & 4.7 & n.s. & n.s. & n.s. & n.s. \\
\hline LT & 0.9 & 2.1 & 3.9 & 2.9 & n.s. & n.s. & n.s. & n.s. \\
\hline RIT & 6.0 & 4.9 & 8.6 & 4.5 & n.s. & n.s. & n.s. & n.s. \\
\hline $\mathrm{RT}$ & 0.6 & 1.0 & 4.6 & 1.1 & n.s. & n.s. & n.s. & n.s. \\
\hline Sum & 10.7 & 12.0 & 24.1 & 13.2 & $\mathrm{n} . \mathrm{s}$ & n.s & n.s & n.s \\
\hline \multicolumn{9}{|l|}{ Occipital } \\
\hline CALC & 19.0 & 27.2 & 28.2 & 29.0 & n.s. & n.s. & n.s. & n.s. \\
\hline LIES & 31.6 & 40.7 & 36.1 & 38.1 & n.s. & n.s. & n.s. & $4.38^{*}$ \\
\hline LSES & 5.7 & 5.9 & 3.6 & 3.6 & $3.99^{*}$ & n.s. & n.s. & n.s. \\
\hline $\mathrm{OP}$ & 38.1 & 51.3 & 46.7 & 39.2 & n.s. & n.s. & n.s. & n.s. \\
\hline RIES & 17.4 & 32.7 & 23.6 & 19.4 & n.s. & n.s. & $3.7^{*}$ & $6.77^{* *}$ \\
\hline RSES & 6.1 & 7.0 & 5.0 & 8.5 & n.s. & n.s. & n.s. & n.s. \\
\hline Sum & 117.7 & 164.9 & 143.2 & 137.7 & n.s. & n.s. & n.s. & n.s. \\
\hline
\end{tabular}

\footnotetext{
${ }_{* *}^{*} p<0.05$.

** $p<0.01$.

*** $p<0.001$.
} 


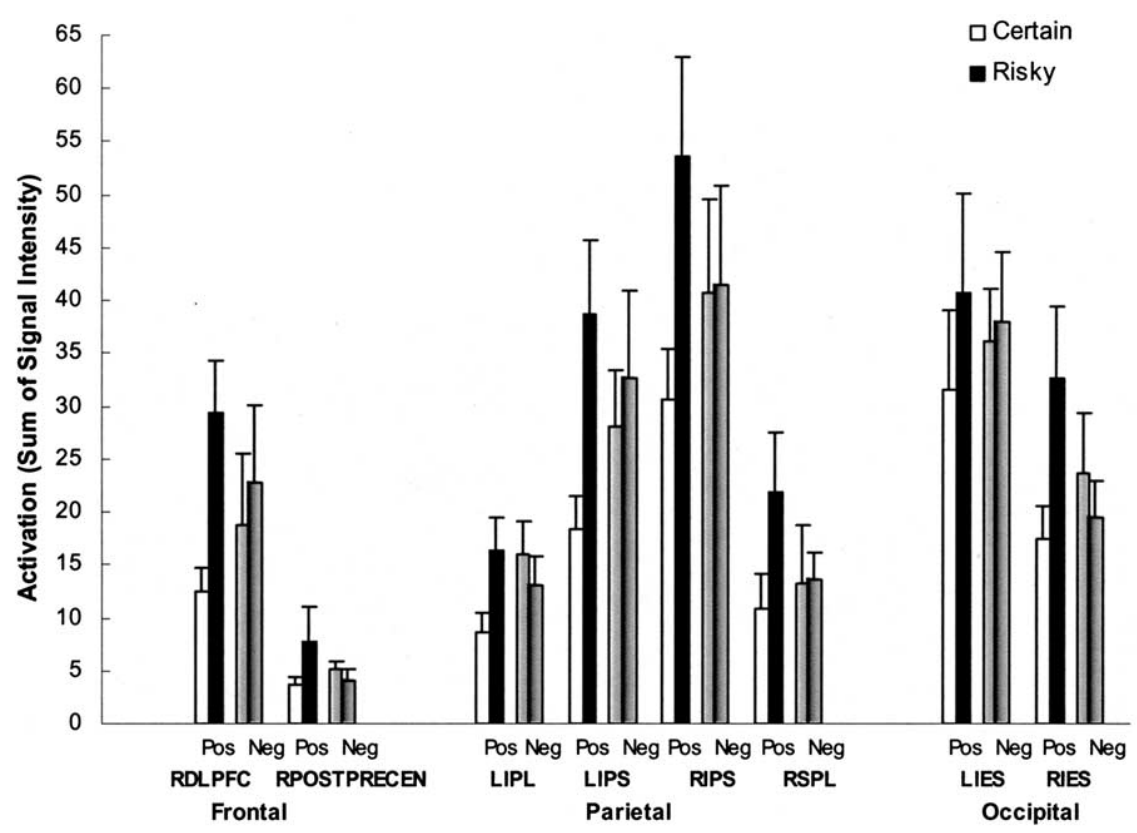

Fig. 3. Frame and risk interaction in specific regions of the frontal (R-DLPFC, R-POSTPRECEN), parietal (L-IPL, L-IPS, R-IPS, R-SPL), and occipital (L-IES, R-IES) areas. Simple effect of selecting risky choices in response to positively framed problems.

cortex (RDLPFC; BA 9 (middle) and 46) and the left and right intraparietal sulcus (LIPS and RIPS, between BA 7 and BA 40) as shown in Table 2. Similar analyses led to no significant difference in response to the positive and negative frames $(p<0.05)$. Fig. 5 graphs the difference in activation between risky and certain choices.

In addition to the activation described above that was measured during deliberation and choice process, we also measured the activation during the reading of the problem (excluding the alternatives). The level of activity (measured with SSI) during reading was much lower (about $85 \%$ lower), and almost always reliably lower $(p<0.05)$ than during choice (see Appendix B).

\section{Discussion}

The behavioral results generated in this study show that participants preferred sure gains to risky ones and risky losses to sure ones, a common empirical result. Individuals also took more time to make decisions framed in terms of losses rather than in terms of gains. The fMRI results demonstrate that the cognitive effort involved in choosing a guaranteed gain is considerably lower than the cognitive effort involved in selecting a risky gain. In contrast, the cognitive effort expended in choosing a guaranteed loss is just as high as that expended in choosing a risky loss. 


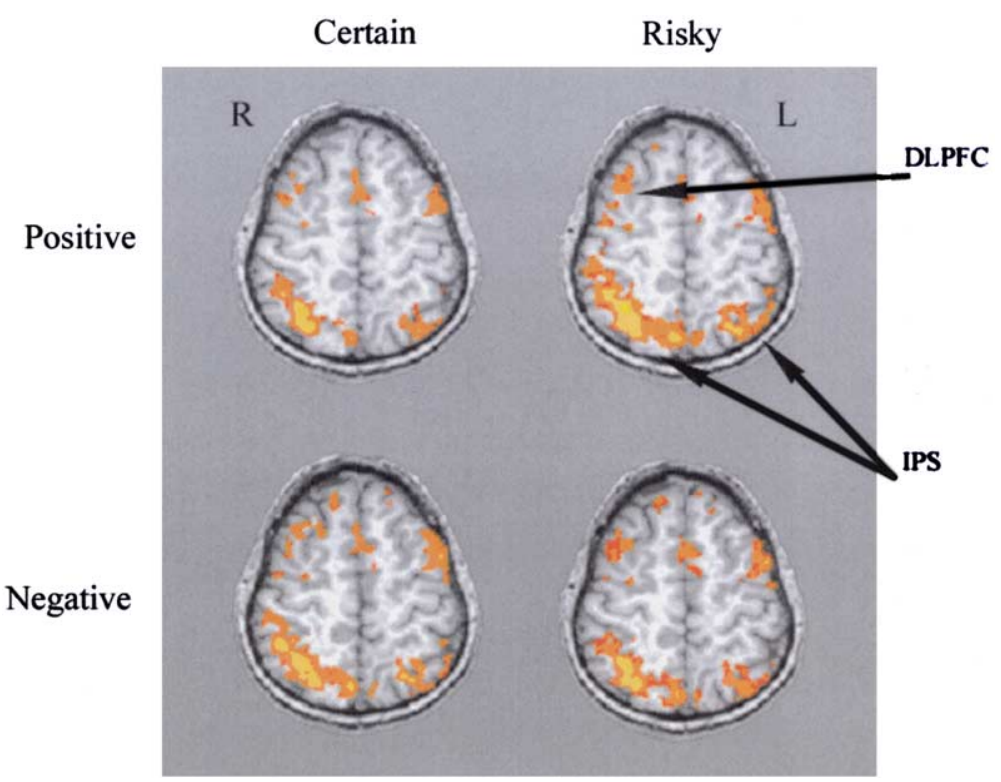

Fig. 4. Threshold fMRI average brain activation images superimposed on a structural image comparing brain activity during the selection of certain versus risky choices in response to positively and negatively framed problems. The Taliarach $Z$ coordinate is 29.250 . Activation in the RDLPFC and IPS regions is higher when risky options are selected instead of certain ones in the positive frame and similarly in the negative frame.

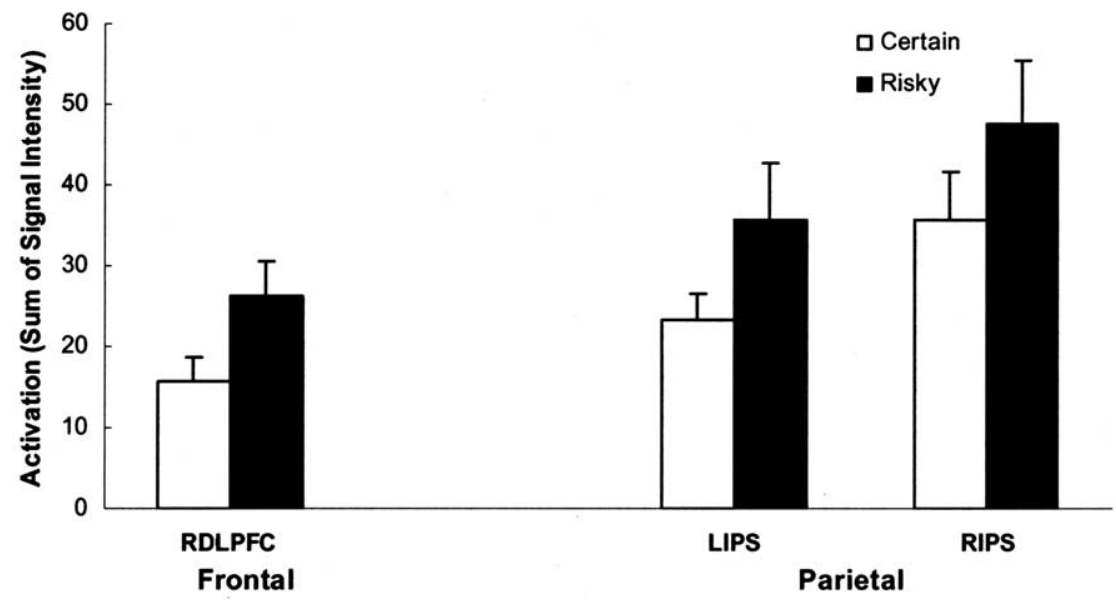

Fig. 5. Higher activation levels in the frontal and parietal lobes of individuals making risky rather than certain choices. Error bars represent $95 \%$ confidence intervals based on the pooled Mse from the corresponding ANOVA. 
These findings support the cognitive-affective tradeoff model of the framing effect proposed in this paper.

The cognitive effort involved in choosing sure or risky alternatives depends on how the alternatives are framed (as gains or losses). fMRI results indicate that the certain choice is considerably less costly (in terms of cognitive effort) than the risky one when individuals choose among options framed as gains. It seems that the tradeoff between minimizing cognitive effort and minimizing feelings of displeasure is easy to resolve in the positive frame. Thus, individuals execute a decision quickly and opt more often for the certain choice.

The differences between brain activity levels in response to positive-certain and positive-risky circumstances were found to be significant in several regions of the frontal and parietal lobes, which suggests that cognitive processes such as working memory and imagery were used more when selecting the risky option than when selecting the certain option. Previous fMRI studies have found that the consideration of large and unlikely rewards leads to more activity in the frontal and parietal cortices of decision makers than does the consideration of small and likely rewards (Rogers et al., 1999). Other studies have demonstrated that activity levels in the orbitofrontal cortex of a problem solver increase with the complexity of the probabilistic demands (Elliott et al., 1999) and the magnitude of the gains and losses under consideration (O'Doherty et al., 2001). Additionally, subcortical activity levels increase with the amount of monetary rewards (Breiter et al., 2001). Because selection of the certain option required very little effort overall to calculate expected value and much less effort than required to evaluate the risky option, participants chose the certain option more often. In so doing, they likely were trying to avoid the cognitive cost involved in evaluating a gain and the emotions involved in imagining an uncertain reward.

In response to negatively framed problems, the tradeoff between minimizing cognitive effort and feelings of displeasure is difficult to perform because both the certain and the risky options involve costs. The risky option again requires the decision maker to calculate its expected value and an emotional cost involved in the possibility of accepting a loss. However, selection of the certain option involves a higher emotional cost by asking the decision maker to accept a guaranteed negative outcome. Decision makers' imagery may influence the emotional effects they experience due to expected outcomes. In particular, researchers have suggested that people are more likely to imagine bad (rather than good) outcomes and how the outcomes could have been improved (Kahneman \& Miller, 1986a). Kosslyn et al. (1996) have analyzed the emotional content of negative versus neutral pictures by evaluating these pictures' effects on neural activity. They observed an increase in visual cortex activity in response to negative imagery relative to neutral imagery. Other studies have used the imagery of diverse emotional situations to determine the magnitude of physiological activity and activation in the ventromedial prefrontal cortex (Bechara et al., 2000). In addition, fMRI studies have shown that situations that conflict with participants' moral values result in higher frontal and parietal activation levels (Greene et al., 2001). Thus, the conflict that results from selecting a sure alternative that demands low cognitive effort but leads to negative outcomes leads to increased activity in frontal and parietal cortices. More specifically, as individuals imagine the 
consequences of a guaranteed negative alternative they experience increased activity in the parietal cortex, and as these individuals consider the conflict between their values and the consequences of the decision, they experience an emotional reaction evidenced by increased activity in the frontal cortex. Thus, the high emotional cost of the negative-certain alternative and the high computational cost of the negative-risky alternative can explain our findings here of similar levels of activation irrespective of participants' choices in response to negatively framed problems. Since participants chose the risky option more often than the certain option in response to such problems, our results suggest that people are more willing to accept a computational rather than an emotional cost, despite the fact that both kinds of costs are similarly demanding in terms of brain activity.

Findings reported in this paper are a good starting point to understand how cognitive and affective processes integrate to determine our choices. Cognitive neuroscientists have aimed to determine how emotion and cognitive control are integrated (Gray, 2001, 2004), and this study shows how both emotion a cognition play a role in making risky choices.

There are however, many questions that arise from the results of this study. The first question relates to the particular localization of emotional and cognitive processes involved in making choices. Gray (2004) suggest that in fact attempting to identify parts of the brain that do specialized jobs might not help obtain a complete understanding of how parts influence each other. Emotion and cognition "intertwine so closely that at times it is impossible to discern which is doing what" (Gray, 2004, p. 46). In this study, choices among positively framed options produced different activation levels depending on whether they were certain or risky, which we attribute to cognitive effort needed to calculate an expected value. The similar activation for choices among negatively framed options is however attributed to two different causes, in the certain choice the feelings of displeasure and in the risky choice to the cognitive effort. The question is, can we disentangle these two "causes" of choice? With the current state of art in cognitive neuroscience, it seems possible to target particular regions of the PFC and subcortical components that may reflect emotional state and at the same time areas of PFC that may be specialized in cognitive control.

A second, perhaps more difficult question to answer refers to the interplay of these two cognitive functions (emotion and cognition) over time. Targeting for the changes of activation over time can perhaps help us understand how emotion and cognition interact and integrate to produce behavior.

\section{Conclusion}

Hundreds of empirical studies have demonstrated the framing effect in many different contexts (Kuhberger, 1997, 1998). Researchers performing these studies often have treated cognition as a black box by focusing on the outcomes rather than on the process by which decisions are made in these contexts. As a result, the subject of how people fall prey to apparently irrational processes such as the framing effect has gone 
largely unaddressed. Our findings offer an explanation based on the underlying zneural processes involved in making choices in response to positively and negatively framed problems. Activity in the frontal and parietal cortices suggests that working memory and imagery mechanisms are involved differentially in choosing risky versus sure options. Individuals considering potential gains display risk aversion that manifests itself as significantly higher brain activity during the selection of risky versus guaranteed responses. Individuals considering potential losses exhibit risk-seeking behavior that manifests itself as comparably more brain activity regardless of the selection of risky or certain prospects.

\section{Acknowledgments}

This research was supported by the Multidisciplinary University Research Initiative Program (MURI), Grant number N00014-01-1-0677. Also, we would like to thank the researchers at the Center for Cognitive Brain Imaging at Carnegie Mellon University for their support.

\section{Appendix A. Brain regions and Brodmann areas}

The cortex is divided into four lobes: frontal, parietal, temporal and occipital.

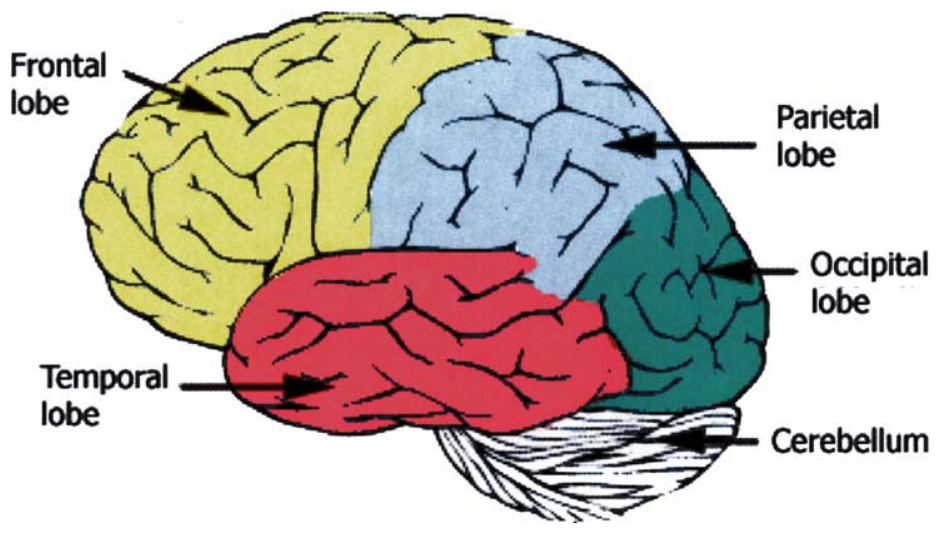

Within each of the regions there are more sub-divisions that are often associated with different cognitive functions. The anatomist Brodmann developed one of the regional classification systems that is in use today based on the architectonic characteristics of the cells in various areas. Fig. 5 shows the Brodmann areas (BAs) in a sagital and mid-sagital view of the brain. For example, BAs 9 and 46 correspond to what is also known as dorsolateral prefrontal cortex (DLPFC) while the BAs 45 and 47 refer to the ventrolateral prefrontal cortex. These areas often become activated in decision making tasks. 

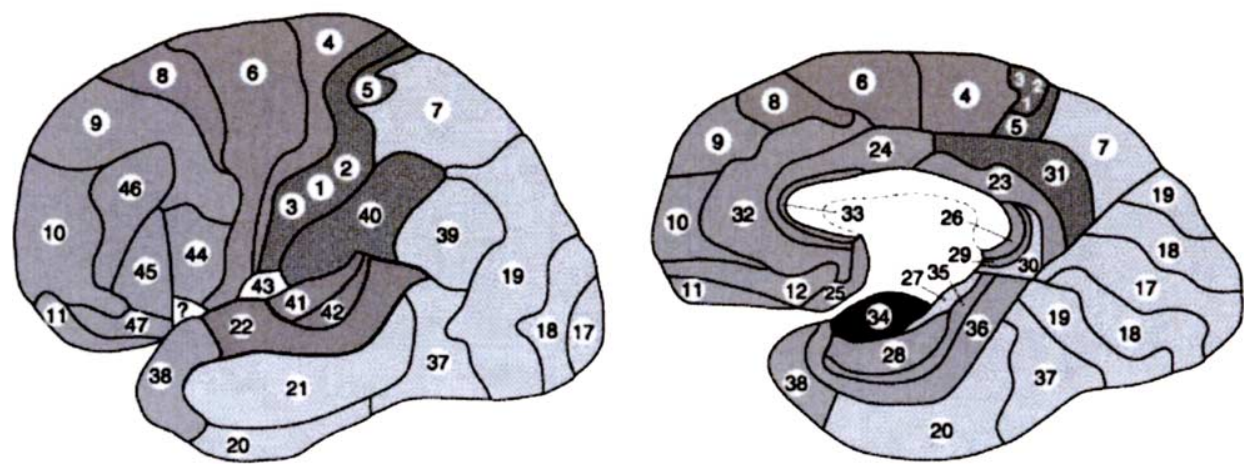

Appendix B. SSI (sum of signal intensity) during reading and choice

\begin{tabular}{|c|c|c|c|}
\hline ROI & Reading & Choice & $F$ \\
\hline \multicolumn{4}{|l|}{ Frontal } \\
\hline LDLPFC & 2.61 & 64.68 & $10.2 * *$ \\
\hline LFEF & 6.14 & 49.35 & $23.67 * * *$ \\
\hline LOPER & 4.45 & 29.23 & $4.05^{*}$ \\
\hline LPOSTPRECEN & 6.38 & 32.02 & $6.75^{*}$ \\
\hline LTRIA & 1.50 & 6.42 & n.s. \\
\hline RDLPFC & 1.47 & 83.56 & $29.99 * * *$ \\
\hline RFEF & 1.41 & 26.33 & $12.84 * * *$ \\
\hline ROPER & 0.63 & 7.21 & $11.92 * *$ \\
\hline RPOSTPRECEN & 3.43 & 20.58 & $5.28 *$ \\
\hline RTRIA & 0.46 & 5.39 & $8.16^{*}$ \\
\hline SMA & 4.46 & 20.31 & $4.28 *$ \\
\hline SMFP & 3.11 & 70.02 & $15.12 * * *$ \\
\hline Sum & 36.04 & 415.11 & $64.01 * * *$ \\
\hline \multicolumn{4}{|l|}{ Parietal } \\
\hline LIPL & 9.15 & 54.06 & $15.69 * * *$ \\
\hline LIPS & 13.49 & 117.93 & $24.0 * * *$ \\
\hline LSGA & 1.05 & 4.65 & n.s. \\
\hline LSPL & 1.55 & 26.83 & $20.23 * * *$ \\
\hline RIPL & 2.42 & 45.98 & $17.08 * * *$ \\
\hline RIPS & 14.79 & 166.53 & $31.44 * * *$ \\
\hline RSGA & 0.00 & 1.59 & $3.88^{*}$ \\
\hline RSPL & 3.20 & 59.40 & $9.61 *$ \\
\hline Sum & 45.65 & 476.97 & $53.23 * * *$ \\
\hline
\end{tabular}


Appendix B (continued)

\begin{tabular}{lrrl}
\hline ROI & Reading & Choice & $F$ \\
\hline Temporal & & & \\
LIT & 3.99 & 19.07 & $6.54^{*}$ \\
LT & 5.71 & 9.83 & n.s. \\
RIT & 2.62 & 23.93 & $4.67^{*}$ \\
RT & 1.99 & 7.25 & n.s. \\
Sum & 14.30 & 60.07 & $12.42^{* * *}$ \\
Occipital & & & \\
CALC & 33.27 & 103.40 & $24.62^{* * *}$ \\
LIES & 26.53 & 146.47 & $16.79^{* * *}$ \\
LSES & 5.12 & 18.72 & $18.70^{* * *}$ \\
OP & 27.51 & 175.25 & $6.43^{*}$ \\
RIES & 16.66 & 93.07 & $23.35^{* * *}$ \\
RSES & 4.14 & 26.60 & $26.05^{* * *}$ \\
Sum & 113.23 & 563.51 & $36.79^{* * *}$ \\
\hline
\end{tabular}

\section{References}

Bandettini, P. A., Wong, E. C., Hinks, R. S., Tokofsky, R. S., \& Hyde, J. S. (1992). Time course EPI of human brain functions during task activation. Magnetic Resonance, 25, 390-397.

Bechara, A., Damasio, H., \& Damasio, A. (2000). Emotion, decision making and the orbitofrontal cortex. Cerebral Cortex, 10(3), 295-307.

Bechara, A., Damasio, H., Tranel, D., \& Damasio, A. (1997). Deciding advantageously before knowing the advantageous strategy. Science, 275, 1293-1294.

Breiter, H. C., Aharon, I., Kahneman, D., Dale, A., \& Shizgal, P. (2001). Functional imaging of neural responses to expectancy and experience of monetary gains and losses. Neuron, 30, 619-639.

Caviness, V. S., Jr., Kennedy, D. N., Bates, J. F., \& Makris, N. (1996). The developing human: A morphometric profile. In R. W. Thatcher \& G. R. Lyon (Eds.). Developmental neuroimaging: Mapping the development of brain and behavior (Vol. xv, pp. 3-14). San Diego: Academic Press, Inc.

Delgado, M. R., Nystrom, L. E., Fissel, C., Noll, D. C., \& Fiez, J. A. (2000). Tracking the hemodynamic responses to reward and punishment in the striatum. The American Physiological Society, 3072-3077.

Eddy, W.F., Fitzgerald, M., Genovese, C.R., Mockus, A., \& Noll, D.C. (1996). Functional imaging analysis software - computational olio a prat. Paper presented at the Computational Statistics, Heidelberg.

Elliott, R., Friston, K. J., \& Dolan, R. J. (2000). Dissociable neural responses in human reward systems. The Journal of Neuroscience, 20(16), 6159-6165.

Elliott, R., Rees, G., \& Dolan, R. J. (1999). Ventromedial prefrontal cortex mediates guessing. Neuropsychologia, 37, 403-411.

Glimcher, P. W. (2003). Decisions, uncertainty and the brain: the science of neuroeconomics. Cambridge, MA: MIT Press.

Gray, J. R. (2001). Emotional modulation of cognitive control: Approach-withdrawal states doubledissociate spatial from verbal two-back task performance. Journal of Experimental Psychology: General, 130, 436-452.

Gray, J. R. (2004). Integration of emotion and cognitive control. Current Directions in Psychological Science, 13(2), 46-48.

Gray, J. R., Braver, T. S., \& Raichle, M. E. (2002). Integration of emotion and cognition in the lateral prefrontal cortex. Proceedings of the National Academy of Sciences USA, 99, 4115-4120. 
Greene, J. D., Sommerville, R. B., Nystrom, L. E., Darley, J. M., \& Cohen, J. D. (2001). An fMRI investigation of emotional engagement in moral judgement. Science, 293, 2105-2108.

Just, M. A., Carpenter, P. A., Keller, T. A., Emery, L., Zajac, H., \& Thulborn, K. R. (2001). Interdependence of nonoverlapping cortical systems in dual cognitive tasks. Neuroimage, 14, 417-426.

Kahneman, D., \& Miller, D. (1986a). Comparing reality to its alternatives. Psychological Review, 153(93), $136-153$.

Kahneman, D., \& Miller, D. (1986b). Norm theory: Comparing reality to its alternatives. Psychological Review, 93, 136-153.

Kahneman, D., \& Tversky, A. (1979). Prospect theory: An analysis of decision under risk. Econometrica, 47(2), 263-291.

Knutson, B., Fong, G. W., Adams, C. M., Varner, J. L., \& Hommer, D. (2001). Dissociation of reward anticipation and outcome with event-related fMRI. NeuroReport, 12(17), 3683-3687.

Kosslyn, S. M., Shin, L. M., Thompson, W. L., McNally, R. J., Rauch, S. L., Pitman, R. K., et al. (1996). Neural effects of visualizing and perceiving aversive stimuli: A PET investigation. NeuroReport, 7, 1569-1576.

Kuhberger, A. (1997). Theoretical conceptions of framing effects in risky decisions. In W. R. Crozier \& O. Svenson (Eds.), Decision making: Cognitive models and explanations. London: Routledge.

Kuhberger, A. (1998). A meta-analysis. Organizational Behavior and Human Decision Processes, 75(1), 23-55.

Levin, I. P., Gaeth, G. J., Schreiber, J., \& Lauriola, M. (2002). A new look at framing effects: Distribution of effect sizes, individual differences, and independence of types of effects. Organizational Behavior and Human Decision Processes, 88(1), 411-429.

Lopes, L. L. (1987). Between hope and fear: The psychology of risk. In L. Berkowitz (Ed.). Advances in experimental social psychology (Vol. 20, pp. 255-295). San Diego: Academic Press.

Maule, A. J. (1995). Framing elaborations and their effects on choice behavior: A comparison across problem isomorphs and subjects with different levels of expertise. In J.-P. Caverni \& M. Bar-Hillel (Eds.). Contributions to decision making (Vol. x, pp. 281-300). Amsterdam, Netherlands: NorthHolland/Elsevier Science Publishers.

Mellers, B., Schwartz, A., \& Ritov, I. (1999). Emotion-based choice. Journal of Experimental Psychology: General, 128(3), 332-345.

Newman, S. D., Just, M. A., \& Carpenter, P. A. (2002). The synchronization of the human cortical working memory network. Neuroimage, 15, 810-822.

O'Doherty, J., Kringelbach, M. L., Rolls, E. T., Hornak, J., \& Andrews, C. (2001). Abstract reward and punishment representations in the human orbitofrontal cortex. Nature Neuroscience, 4(1), 95-102.

Paulus, M. P., Hozack, N., Zauscher, B., McDowell, J. E., Frank, L., Brown, G. G., et al. (2001). Prefrontal, parietal, and temporal cortex networks underlie decision-making in the presence of uncertainty. Neuroimage, 91-100.

Payne, J. W., Bettman, J. R., \& Johnson, E. J. (1993). The adaptive decision maker. New York, NY: Cambridge University Press.

Rademacher, J., Galaburda, A. M., Kennedy, D. N., Filipek, P. A., \& Caviness, V. S. Jr., (1992). Human cerebral cortex: Localization, parcellation, and morphonetry with magnetic resonance imaging. Journal of Cognitive Neuroscience, 4, 352-374.

Reyna, V. F., \& Brainerd, C. J. (1991). Fuzzy-trace theory and framing effects in choice. Journal of Behavioral Decision Making, 4, 249-262.

Rogers, R. D., Owen, A. M., Middleton, H. C., Williams, E. J., Pickard, J. D., Sahakian, B. J., et al. (1999). Choosing between small, likely rewards and large, unlikely rewards activates inferior and orbital prefrontal cortex. The Journal of Neuroscience, 20(19), 9029-9038.

Tversky, A., \& Kahneman, D. (1981). The framing of decisions and the psychology of choice. Science, 211, $453-458$.

Xiong, J., Rao, S., Gao, J.-H., Woldor, M., \& Fox, P. T. (1998). Evaluation of hemispheric dominance for language using functional MRI: A comparison with positron emission tomography. Human Brain Mapping, 6, 42-58. 